\title{
Fatores Associados à Sintomatologia Depressiva em Idosos
}

\author{
Claudia Daiane Trentin Lampert ${ }^{1}$, Vinícius Renato Thomé Ferreira \\ Faculdade IMED, Passo Fundo-RS, Brasil
}

\section{RESUMO}

A presença de sintomas depressivos em idosos associa-se a diversos fatores e pode resultar em pior qualidade de vida. Este estudo investigou a presença e fatores associados à sintomatologia depressiva em idosos. Participaram 112 idosos, média de idade de 67,07 anos $(D P=9,11)$, atendidos nos serviços de atenção básica de saúde. Os instrumentos utilizados foram: Questionário sociodemográfico; Miniexame do Estado Mental; Índice de Katz; Escala CES-D e Levantamento da Intensidade de Sintomas Depressivos (LIS-D). Os resultados indicam para a presença de sintomas depressivos em 35,7\% dos participantes, sendo que as mulheres apresentaram mais sintomas e maior comprometimento da capacidade funcional. Morar sozinho, maior dependência funcional e déficits cognitivos foram associados à presença de sintomas depressivos. O conhecimento da presença e dos fatores associados aos sintomas depressivos em idosos pode promover ações voltadas para a qualidade de vida nesta população.

Palavras-chave: depressão; envelhecimento; fatores de risco; saúde mental.

\section{ABSTRACT - Factors Associated with Depressive Symptomatology in the Elderly}

The presence of depressive symptoms in the elderly is associated with several factors and may result in poorer quality of life. This study investigated the factors and presence associated with depressive symptomatology in the elderly. A total of 112 elderly people participated in the study, with a mean age 67.07 years $(S D=9.11)$, attended for basic health care services. The instruments applied included a Socio-demographic questionnaire; Mini-Mental State Examination; the Katz Index; the CES-D Scale, and the Intensity of Depressive Symptoms Survey (LIS-D). Results indicate the presence of depressive symptoms in 35.7\% of the participants, with the women presenting more symptoms and greater impairment of functional capacity. Living alone, greater functional dependence and cognitive deficits were associated with the presence of depressive symptoms. Knowledge of the presence and factors associated with depressive symptoms in the elderly can promote actions focused on the quality of life in this population.

Keywords: depression; aging; risk factors; mental health.

\section{RESUMEN - Factores Asociados a Sintomatología Depresiva en Ancianos}

La presencia de síntomas depresivos en ancianos está asociada a diversos factores y como resultado pueden pasar a tener peor calidad de vida. Este estudio investigó la presencia y factores asociados a la sintomatología depresiva en ancianos. Participaron 112 ancianos, con edad media de 67,07 años $(D P=9,11)$, atendidos en los servicios de atención básica de salud. Los instrumentos utilizados fueron: Cuestionario sociodemográfico; Mini Examen de Estado Mental; Índice de Katz; Escala CES-D y Levantamiento de la Intensidad de Síntomas Depresivos (LIS-D). Se constató la presencia de síntomas depresivos en 35,7\% de los participantes, siendo las mujeres las que presentaron más síntomas y mayor comprometimiento de capacidad funcional. Vivir solos, mayor dependencia funcional y déficits cognitivos fueron asociados a la presencia de síntomas depresivos. El conocimiento de la presencia y de factores asociados a los síntomas depresivos en ancianos, puede promover acciones relacionadas con la calidad de vida en esta población.

Palabras clave: depresión; envejecimiento; factores de riesgo; salud mental.

Os sintomas depressivos são um problema de magnitude mundial, afetando uma estimativa de $350 \mathrm{mi}-$ lhões de pessoas, desde jovens a idosos. Um inquérito de saúde mental realizado em 17 países constatou que, em média, cerca de uma em cada vinte pessoas, ou seja, 5\%, relataram ter um episódio depressivo no ano anterior, e uma em cada cinco pessoas desenvolvem transtorno depressivo maior durante algum momento de sua vida.
As previsões epidemiológicas apontam que, em 2020, os quadros depressivos serão a segunda principal causa de incapacidade mundo e, em 2030, espera-se que os sintomas depressivos sejam o principal fator relacionado com as doenças (Organização Mundial de Saúde, 2001; World Health Organization, 2012).

Vários fatores estão relacionados para o surgimento de sintomas depressivos: biológicos, genéticos, 
psicossociais e distorções cognitivas, que podem afetar as pessoas em diferentes momentos da vida (OMS, 2001). Conforme Smith, Haedtke e Shibley (2015), a população idosa merece especial atenção, visto o crescente número de idosos que apresentam fatores de risco para a depressão, é necessário maior foco de atenção em estudos e métodos de rastreio e detecção da depressão, e tratamento e prevenção, nessa população quevai continuar a crescer mundialmente.

Nos idosos, o quadro depressivo se distingue de outras faixas etárias em virtude das diferenças de sintomatologia e circunstâncias existenciais específicas da idade, apresentando uma diminuição da resposta emocional, ausência de afeto positivo e predomínio de sintomas, como diminuição do sono, perda de prazer nas atividades habituais, ruminações sobre o passado e perda de energia. Desse modo, os sintomas depressivos apresentam-se mais como queixas de sintomas somáticos, cognitivos e hipocondríacos do que de humor deprimido ou culpa (Smith, Haedtke, \& Shibley, 2015; Taylor, 2014; Unützer \& Park, 2012). A presença de sintomas que configurem um quadro depressivo no idoso pode afetar sua qualidade de vida, interferir nas condições de saúde, funcionalidade física e mental, afetividade, motivação, independência e autonomia, bem como aumentar o risco de suicídio e atuar como fator de risco para o desenvolvimento de um processo demencial. Ainda, quando associadas a doenças clínicas, os sintomas depressivos agravam essas enfermidades, sobretudo aquelas que imprimem sofrimento prolongado, elevando os índices de hospitalizações e mortalidade (Batistone \& Neri, 2007; Cavalcante, Minayo, \& Mangas, 2013; Smith et al., 2015).

A incidência de transtornos depressivos em idosos na comunidade apresenta significativa prevalência, variando entre $4,8 \%$ e $14,6 \%$, podendo chegar a $22 \%$ em idosos institucionalizados. No Brasil, alguns estudos epidemiológicos de base populacional apontam uma prevalência de sintomas depressivos, variando entre $18 \%$ a $23,9 \%$ em idosos residentes na comunidade (Borges, Benedetti, Xavier, \& D’Orsi, 2013; Lima, Silva, \& Ramos, 2009; Bretanha et al., 2015; Pinho, Custódio, \& Makdisse, 2009). As diferenças nos índices de prevalência de sintomas depressivos podem variar em função das características peculiares das pessoas em diferentes contextos, número de indivíduos estudados, e dos vários métodos utilizados, diferentes pontos de corte para diagnosticar ou realizar rastreios para a sintomatologia, muitas vezes dificultando a comparabilidade de resultados pontuais (Coutinho, Hamdam, \& Baptista, 2016; Lima et al., 2009; Hoffmann, Ribeiro, Farnese, \& Lima, 2010).

Os fatores sociodemográficos e de saúde associados à sintomatologia depressiva em idosos relatados na literatura incluem: sexo feminino, idade avançada, condição marital, baixa escolaridade, condição socioeconômica desfavorável, piores condições de moradia, baixo suporte social, presença de eventos de vida estressores, histórico psiquiátrico prévio, presença de comorbidades psiquiátricas, características de personalidade, distúrbios do sono, déficits cognitivos, doenças crônicas e agudas, comorbidades orgânicas, gravidade da doença, pior autopercepção de saúde, limitação funcional e presença de dor (Alvarenga, Oliveira, Faccenda, Cerchiari, \& Amendola, 2010; Batistone, Neri, \& Cupertino, 2010; Borges et al., 2013, Pinho et al., 2009, Sass, Gravena, Pilger, Mathias, $\&$ Marcon, 2012).

No atendimento à saúde na atenção básica, a identificação de sintomas depressivos na população idosa ainda é precária, devido ao despreparo de alguns profissionais e o uso de escalas de mensuração e rastreio sem confiabilidade. Em muitos casos, os profissionais de saúde veem os sintomas depressivos como manifestações normais decorrentes do processo de envelhecimento ou os confundem com ansiedade e tristeza (Fernandes, Nascimento, \& Costa, 2009; Smith et al., 2015). A adequada identificação dos sintomas depressivos derivado de um satisfatório treinamento pelos profissionais da saúde é fundamental para o atendimento e tratamento da população idosa, não se focando apenas na saúde biológica, mas incluindo as questões psicológicas.

Nesse sentido, torna-se necessário a realização de estudos que identifiquem a ocorrência de sintomas depressivos na população idosa e os fatores envolvidos. Ainda, a utilização de instrumentos de rastreio adequados pode subsidiar conhecimentos que pautem ações voltadas para o tratamento, promoção de saúde e ofertas de cuidado na atenção básica. Ante ao exposto, este estudo objetivou investigar a presença de sintomas depressivos em idosos, identificando sua prevalência e quais os fatores psicossociais que podem estar associados a essa sintomatologia.

\section{Método}

\section{Participantes}

A amostra foi composta por 112 idosos, com idade igual ou superior a 60 anos, homens e mulheres, alfabetizados, de diferentes níveis socioeconômicos e atendidos nos serviços de atenção básica de um município no interior do Rio Grande do Sul. Os critérios de exclusão utilizados foram idade inferior a 60 anos e não possuir condições de compreender e responder aos instrumentos averiguado pela pontuação igual ou menor a 20 pontos no Mini-exame do Estado Mental - MEEM (Bertolucci, Brucki, Campacci, \& Juliano, 1994).

\section{Instrumentos}

Questionário de caracterização sociodemográfica e condições de saúde, elaborado pelos autores, foi constituído por 16 questões fechadas sobre idade, gênero, etnia, estado civil, escolaridade, renda familiar, profissão/ ocupação, religião, eventos de vida estressores e condições de saúde. 
Escala de Independência em Atividades da Vida Diária de Katz - Índice de Katz (Lino, Pereira, Camacho, Ribeiro Filho, \& Buksman, 2008). Utilizada para avaliar a capacidade funcional para o desempenho de algumas atividades cotidianas básicas, como banhar-se, vestir-se, ir ao banheiro, transferências da cama para cadeira e vice-versa, controle sobre os esfíncteres e alimentação sem assistência, indicando o desempenho do indivíduo nas atividades de autocuidado. É um dos instrumentos mais utilizados para avaliar Atividades de Vida Diária (AVD) e apresenta em sua classificação o Index, que varia de 0 (totalmente independente) a 6 (totalmente dependente). A classificação considerou: 0 - quando independente em todas as seis funções; 1 - independente em cinco funções e dependente em uma função; 2 - independente em quatro funções e dependente em duas; 3 - independente em três funções e dependente em três; 4 - independente em duas funções e dependente em quatro; 5 - independente em uma função e dependente em cinco funções; 6 - dependente em todas as seis funções. A avaliação é baseada no real desempenho, não na habilidade que o indivíduo julgue ser capaz. No Brasil, o processo de adaptação transcultural da escala obteve concordância de 78,9\% e alfa de Cronbach de 0,80 a 0,92 (Lino et. al., 2008).

Miniexame do Estado Mental - MEEM (Bertolucci et al., 1994). Objetivou avaliar aspectos cognitivos e verificar os critérios de inclusão e exclusão da amostra. Composto por questões agrupadas em sete categorias, com um escore total de 0 a 30 pontos. Foram utilizados os pontos de corte do estudo de Brucki, Nitrini, Caramelli, Bertolucci e Okamoto (2003), a saber: baixa escolaridade (1 a 4 anos) - 20 pontos; média escolaridade (4 a 8 anos) - 24 pontos; alta escolaridade (acima de 8 anos) -26 pontos. O instrumento apresentou sensibilidade de $97,5 \%$ para analfabetos; $96,6 \%$ para baixa e média escolaridade e $95,6 \%$ para alta escolaridade (Bertolucci et al., 1994).

Center for Epidemiological Studies - CES-D (Radlof, 1977, adaptada por Silveira \& Jorge, 1998). Consiste em uma escala de rastreio de sintomas depressivos, composta por 20 itens, que comportam avaliação da frequência de sintomas depressivos vividos na semana anterior à entrevista $(0=$ nunca ou raramente, 2 $=$ às vezes, $3=$ maioria das vezes ou sempre). $\mathrm{O}$ escore $\mathrm{fi}-$ nal varia de 0 a 60 pontos. O ponto de corte utilizado para identificar presença de sintomas depressivos é 17 pontos; o alfa de Cronbach do teste é de 0,85). Estudos com o CES-D em população idosa demonstraram bons índices de consistência interna e de validade de construto, assim como mostrou reprodutibilidade teste-reteste (Batistone et al., 2010).

Levantamento da Intensidade de Sintomas Depressivos (LIS-D). É um instrumento de autoavaliação que visa identificar a intensidade de sintomas depressivos. O LIS-D está baseado no referencial teórico da psicologia cognitivo-comportamental, e avalia sintomas depressivos em quatro dimensões (sintomas afetivos, somáticos, de pensamento e comportamentais) e tríade cognitiva (visão de si, visão do outro e visão do futuro). $\mathrm{O}$ instrumento encontra-se em processo de pesquisa e validação (Ferreira, 2012).

\section{Procedimentos}

Após a autorização da secretaria de saúde do município onde a pesquisa foi realizada para a aplicação dos instrumentos nos serviços de saúde do município, e por aprovação por Comitê de Ética em Pesquisa (parecer 1.011.187), deu-se início à coleta de dados. Os participantes foram entrevistados em quatro serviços de saúde do município, duas Estratégias de Saúde da Família (ESF) e dois Centros de Atendimento Integral de Saúde (CAIS) que abrangeram diferentes regiões geográficas do município. A aplicação dos instrumentos foi individual, em sala apropriada e com um tempo de duração de aproximadamente uma hora. Após explicar os objetivos do estudo e verificado os critérios de exclusão, os idosos que consentiram participar da pesquisa assinaram o Termo de Consentimento Livre e Esclarecido e responderam aos instrumentos, iniciando pelo questionário de caracterização sociodemográfico, seguido da Escala Katz e das escalas CES-D e LIS-D.

Os dados foram analisados quantitativamente, com uma análise descritiva das variáveis e uma estatística inferencial, estudando correlações e efetuando testes de hipóteses com testes não paramétricos, como MannWhitney. Para a análise de variância dos fatores sociodemográficos e sintomas depressivos, utilizou-se o teste de Kruskal-Wallis. As correlações entre os instrumentos foram verificadas por meio da correlação de Spearman, adotando os parâmetros de Dancey e Reidy (2013) para a verificação da força da correlação. O nível de significância adotado foi de $p \leq 0,05$ e intervalo de confiança de $95 \%$.

\section{Resultados}

$\mathrm{Na}$ descrição dos resultados, é exposta, primeiramente, a caracterização sociodemográfica da população em estudo. Em seguida, são apresentados os resultados dos participantes nos instrumentos e avaliação da diferença entre homens e mulheres. Na sequência, demonstram-se as correlações entre os instrumentos o fator idade. A Tabela 1 apresenta a caracterização sociodemográfica da população em estudo.

Os idosos participantes do estudo tinham entre 60 e 92 anos, com média de 67,07 anos $(D P=9,11)$, sendo a maior proporção do sexo feminino $(71,43 \%)$. Quanto a escolaridade, $59,82 \%$ possuíam o ensino fundamental incompleto e destes $56,7 \%$ tinham até quatro anos de estudo. Em relação ao estado civil, $42,86 \%$ eram casados e $38,39 \%$ eram viúvos; a maioria referiu morar com o 
cônjuge $(43,75 \%)$ ou sozinho $(27,68 \%)$. No tocante à profissão/ocupação, 74,11\% dos idosos eram aposentados e $22,32 \%$ estavam trabalhando em atividades, como diarista, costureira, motorista, vendas e comércio. A renda familiar predominante foi igual ou inferior a dois salários mínimos (51,79\%). Com relação às doenças autorreferenciadas, as mais prevalentes foram hipertensão arterial (46,42\%), seguido de diabetes mellitus $(15,17 \%)$ e doenças cardíacas $(14,29 \%)$; apenas $25,89 \%$ dos idosos referiram não ter nenhuma doença.

Tabela 1

Caracterização Sociodemográfica da População em Estudo (n=112)

\begin{tabular}{|c|c|c|c|}
\hline Item & Variável & Frequência & $\%$ \\
\hline \multirow[t]{2}{*}{ Sexo } & Feminino & 80 & 71,4 \\
\hline & Masculino & 32 & 28,5 \\
\hline \multirow[t]{6}{*}{ Escolaridade } & Fundamental incompleto & 67 & 59,8 \\
\hline & Fundamental completo & 15 & 13,3 \\
\hline & Médio incompleto & 2 & 1,7 \\
\hline & Médio completo & 21 & 18,7 \\
\hline & Superior incompleto & 1 & 0,8 \\
\hline & Superior completo & 6 & 5,3 \\
\hline \multirow[t]{4}{*}{ Estado civil } & Solteiro & 13 & 11,6 \\
\hline & Casado/Un. est. & 48 & 42,8 \\
\hline & Separado/divorciado & 8 & 7,1 \\
\hline & Viúvo & 43 & 38,3 \\
\hline \multirow[t]{3}{*}{ Religião } & Cristã & 109 & 97,3 \\
\hline & Espiritualista & 2 & 1,7 \\
\hline & Mórmon & 1 & 0,8 \\
\hline \multirow[t]{3}{*}{ Profissão/ocupação } & Aposentado & 83 & 74,1 \\
\hline & Pensionista & 4 & 3,5 \\
\hline & Empregado/autônomo & 25 & 22,3 \\
\hline \multirow[t]{5}{*}{ Renda familiar } & Entre 0 e 2 SM & 58 & 51,7 \\
\hline & Entre 3 e 4 SM & 29 & 25,8 \\
\hline & Entre 5 e 6 SM & 19 & 16,9 \\
\hline & Entre 7 e 10 SM & 5 & 4,4 \\
\hline & Entre 11 e 15 SM & 1 & 0,8 \\
\hline \multirow[t]{5}{*}{ Reside com } & Cônjuge & 49 & 43,7 \\
\hline & Filho/a & 29 & 25,8 \\
\hline & Neto/a & 2 & 1,7 \\
\hline & Sozinho/a & 31 & 27,6 \\
\hline & Não respondeu & 1 & 0,8 \\
\hline \multirow[t]{8}{*}{ Referência de doenças } & Sem doenças & 29 & 25,8 \\
\hline & Hipertensão Arterial & 52 & 46,4 \\
\hline & Diabete Mellitus & 17 & 15,1 \\
\hline & Cardíaca & 16 & 14,2 \\
\hline & Articulações & 8 & 7,1 \\
\hline & Oftalmológica & 2 & 1,7 \\
\hline & Hormonal & 3 & 2,6 \\
\hline & Circulatória & 2 & 1,7 \\
\hline
\end{tabular}

Nota. Elaborada pelos autores

A comparação da diferença do desempenho dos homens e mulheres nos instrumentos apontou para uma boa independência e manutenção de suas capacidades funcionais. A comparação entre homens e mulheres apontou para uma diferença estatisticamente significativa
$(U=1079, p=0,032)$, indicando que as idosas apresentam maiores prejuízos da manutenção da independência para as AVDs, com maior comprometimento da capacidade funcional. Em relação às funções cognitivas, a média total no MEEM foi de 24,7, estando próxima ao ponto 
de corte para indicativo de prejuízo cognitivo (24 pontos ou menos indica a presença de prejuízo). Contudo, considerando a baixa escolaridade apresentada pelos idosos, pode-se inferir para a preservação das capacidades cognitivas.

O CES-D sugere como ponto de corte para a presença de sintomas depressivos valores iguais ou superiores a 17 pontos. Em relação ao desempenho geral, a média da pontuação na CES-D na amostra estudada esteve pouco abaixo desse indicador $(M=16,14, D P=14,15)$ e, mesmo não obtendo diferença estatisticamente significativa, as mulheres $(M=17,6, D P=15)$ apresentaram mais sintomas depressivos quando comparados aos homens $(M=12,3, D P=10,8)$. Foi observado um índice de $35,7 \%$ de participantes com pontuação igual ou superior a 17 pontos $(n=40)$. Com relação aos resultados no LIS-D, os idosos obtiveram escores médios de 31 pontos $(D P=27,7)$. O LIS-D no momento ainda não apresenta pontos de corte para sintomas depressivos, por estar em processo de validação, mas as maiores pontuações referiram a maior presença de sintomas. Os sintomas somáticos apresentaram maior pontuação $(M=8, D P=5,4)$, sendo que as mulheres apresentaram escores mais elevados, além de maior comprometimento nas AVDs quando comparadas aos homens $(U=1079, p=0,03)$.

Considerando as associações entre fatores sociodemográficos e sintomas depressivos, o arranjo familiar de morar sozinho versus morar com alguém apontou diferenças estatisticamente significativas na visão de si do LIS-D ( $U=965,5 ; p=0,051)$ e visão do futuro do LIS-D $(U=1040 ; p=0,034)$ com mais prejuízo para quem mora sozinho. Também houve diferença estatisticamente significativa no CES-D, apontando mais sintomas depressivos no grupo que mora sozinho $(U=917 ; p=0,028)$. Em relação à idade e escolaridade, não houve associações significativas, não sendo fatores diretamente associados à presença dos sintomas depressivos nos idosos entrevistados. Esses resultados estão apresentados na Tabela 2.

Tabela 2

Desempenho dos Participantes nos Instrumentos e Avaliação da Diferença entre Homens e Mulheres

\begin{tabular}{|c|c|c|c|c|c|c|c|c|c|c|c|}
\hline \multirow[b]{2}{*}{ Instrumento } & \multicolumn{3}{|c|}{ Total } & \multicolumn{3}{|c|}{ Mulheres } & \multicolumn{3}{|c|}{ Homens } & \multicolumn{2}{|c|}{ Mann-Whitney } \\
\hline & Média & DP & $\begin{array}{c}\text { IC } \\
(95 \%)\end{array}$ & Média & $\mathrm{DP}$ & $\begin{array}{c}\text { IC } \\
(95 \%)\end{array}$ & Média & DP & $\begin{array}{c}\mathrm{IC} \\
(95 \%)\end{array}$ & $\mathrm{U}$ & $\begin{array}{c}p \\
\text { unicaudal }\end{array}$ \\
\hline Katz & 0,19 & 0,59 & 0,06 & 0,25 & 0,68 & 0,08 & 0,03 & 0,18 & 0,03 & 1079,00 & 0,032 \\
\hline MEEM & 24,78 & 3,14 & 0,30 & 24,59 & 3,40 & 0,38 & 25,25 & 2,36 & 0,42 & 1161,50 & 0,443 \\
\hline CES-D & 16,14 & 14,15 & 1,34 & 17,65 & 15,06 & 1,68 & 12,38 & 10,87 & 1,92 & 1029,50 & 0,106 \\
\hline LIS-D afeto & 5,42 & 5,51 & 0,52 & 5,68 & 5,80 & 0,65 & 4,78 & 4,73 & 0,84 & 1220,00 & 0,695 \\
\hline LIS-D pensamento & 5,06 & 5,18 & 0,49 & 5,60 & 5,64 & 0,63 & 3,72 & 3,53 & 0,62 & 1095,50 & 0,230 \\
\hline LIS-D comportamento & 2,99 & 4,15 & 0,39 & 3,30 & 4,20 & 0,47 & 2,22 & 3,99 & 0,71 & 1074,50 & 0,166 \\
\hline LIS-D somático & 8,01 & 5,49 & 0,52 & 8,58 & 5,67 & 0,63 & 6,59 & 4,79 & 0,85 & 1017,00 & 0,088 \\
\hline LIS-D visão de si & 3,61 & 4,60 & 0,43 & 3,81 & 4,83 & 0,54 & 3,09 & 3,99 & 0,70 & 1222,00 & 0,699 \\
\hline LIS-D visão do mundo & 4,24 & 4,56 & 0,43 & 4,16 & 4,78 & 0,53 & 4,44 & 4,02 & 0,71 & 1158,00 & 0,422 \\
\hline LIS-D visão do futuro & 2,63 & 3,97 & 0,37 & 2,80 & 3,97 & 0,44 & 2,22 & 3,98 & 0,70 & 1139,00 & 0,321 \\
\hline LIS-D total & 31,96 & 27,74 & 2,62 & 33,93 & 29,43 & 3,29 & 27,06 & 22,65 & 4,00 & 1147,50 & 0,393 \\
\hline
\end{tabular}

Nota. Elaborada pelos autores

A seguir, a Tabela 3 apresenta a correlação entre os instrumentos e o fator idade. Como observado, entre os resultados obtidos por meio da correlação entre os instrumentos, observa-se correlação significativa, porém de baixa magnitude, entre idade e funções cognitivas $(r=-0,34, p \leq 0,001)$, indicando que quanto maior a idade, mais prejudicadas estão as funções cognitivas do idoso. A escala CES-D apresentou correlações baixa e positivas com a capacidade para realização das AVD $(r=0,27$, $p<0,01)$ baixa e negativas quanto às funções cognitivas $(r=-0,32, p \leq 0,001)$. Ou seja, quanto maior a dependência funcional e prejuízos cognitivos apresentados pelo idoso, haverá um sutil maior risco de sintomas depressivos.

A pontuação total do LIS-D apresentou baixa correlação positiva com o índice de Katz $(r=0,32, p<0,001)$ e nas dimensões afeto $(r=0,27, p=0,005)$, pensamento $(r=0,21, p=0,02)$ comportamento $(r=0,26, p=0,006)$, somáticos $(r=0,31, p<0,001)$, visão de si $(r=0,18, p=0,05)$ visão do mundo $(r=0,28, p=0,002)$ e visão do futuro $(r=0,21, p=0,03)$. Esses resultados sugerem que quanto maior a dependência funcional apresentada pelo idoso, maior o aumento dos sintomas depressivos afetivos, do pensamento, comportamentais e somáticos e uma visão de si, do mundo e do futuro mais pessimista. Ainda, o LIS-D total apresentou correlações negativas, porém fracas, com o MEEM $(r=-0,25, p=0,007)$, e nas dimensões afeto $(r=-0,25, p=0,008)$, comportamento $(r=-0,29$, $p=0,002)$, pensamento $(r=-0,21, p=0,02)$, visão de si $(r=0,22, p=0,02)$ e visão do mundo $(r=0,18, p=0,006)$. Os prejuízos cognitivos nos idosos relacionam-se ao 
aumento de sintomas depressivos relacionados ao afeto, comportamento, pensamento e uma visão de si e do mundo negativa. Em relação aos instrumentos de aferição de sintomas depressivos utilizados, observou-se forte correlação entre a escala CES-D e o LIS-D total $(r=0,81$, $p<0,001)$ e entre as CES-D e as todas as oito dimensões do LIS-D, apontando para a satisfatória validade de construto da escala LIS-D.

Tabela 3

Correlação entre os Instrumentos e Fator Idade

\begin{tabular}{lcccc}
\hline & Idade & Katz & Mini Mental & CES-D \\
\hline Katz & $0,18(0,06)$ & $1(-)$ & & \\
Mini Mental & $-0,34(<0.001)$ & $-0,18(0,06)$ & $1(-)$ & $1(-)$ \\
CES-D & $0,09(0,32)$ & $0,27(<0,01)$ & $-0,32(<0,001)$ & $-0,25(0,008)$ \\
LIS-D afeto & $0,030(0,75)$ & $0,27(0,005)$ & $-0,29(0,002)$ & $0,62(<0,001)$ \\
LIS-D comportamento & $-0,03(0,72)$ & $0,26(0,006)$ & $-0,21(0,02)$ & $0,64(<0,001)$ \\
LIS-D pensamento & $0,00(0,92)$ & $0,21(0,02)$ & $-0,09(0,32)$ & $0,64(<0,001)$ \\
LIS-D somáticos & $0,01(0,89)$ & $0,31(<0,001)$ & $-0,22(0,02)$ & $0,62(<0,001)$ \\
LIS-D visão de si & $0,08(0,37)$ & $0,18(0,05)$ & $-0,18(0,006)$ & $0,55(<0,001)$ \\
LIS-D visão do mundo & $-0,02(0,80)$ & $0,28(0,002)$ & $-0,08(0,38)$ & $0,50(<0,001)$ \\
LIS-D visão do futuro & $-0,09(0,33)$ & $0,21(0,03)$ & $-0,25(0,007)$ & $0,81(<0,001)$ \\
LIS-D total & $0,03(0,75)$ & $0,32(<0,001)$ & & \\
\hline
\end{tabular}

Nota. Elaborada pelos autores

\section{Discussão}

Com relação às características sociodemográficas dos idosos entrevistados, nota-se o predomínio de indivíduos do sexo feminino, com baixa escolaridade e renda familiar, resultados semelhantes a grande parte dos estudos com idosos (Batistone et al., 2010; Bretanha et al., 2015; Borges et al., 2013; Hoffmannet al., 2010, Sass et al., 2012, Oliveira et al., 2012). Esses dados condizem com a tendência nacional que aponta para a feminilização da velhice e para a realidade socioeconômica da maioria dos idosos, principalmente os aposentados (Brasil, 2002). As doenças autorreferidas mais prevalentes neste estudo foram hipertensão arterial, diabetes mellitus e doenças cardíacas, concordando com outros estudos conduzidos com essa população (Fernandes et al., 2009; Sass et al., 2012).

As médias gerais nas pontuações nas escalas CES-D e LIS-D não alcançaram valores significativos para sintomas depressivos; contudo, a presença de sintomas depressivos indicada por escores do CES-D superior a 17 pontos em $35,7 \%$ dos idosos pode ser considerada alta em relação à população geral, a qual é de aproximadamente 13\% (Pinho et al., 2009). Não há concordância sobre a epidemiologia dos sintomas depressivos em populações de idosos, que pode ser atribuído a diferentes metodologias ou instrumentos; a literatura aponta para valores menores de prevalência, como $18 \%$ a $24 \%$ (Borges et al., 2013; Bretanha et al., 2015; Lima et al., 2009; Oliveira et al., 2012), enquanto que outros estudos revelam valores mais elevados, variando de $30 \%$ a 52\% (Fernandes et al., 2009; Alvarenga et al., 2010; Sass et al., 2012). Os valores encontrados sinalizam para a importância de investigar sintomas depressivos em idosos atendidos pela atenção básica.

Mulheres apresentara mais sintomas somáticos e maiores índices de dependência na realização das AVDs, o que pode estar associado a maior presença de sintomas depressivos nesse grupo. Esse achado está em concordância com outros estudos sobre sintomas depressivos em idosos (Bretanha et al., 2015; Borges et al., 2013; Fernandes et al., 2009; Lima et al., 2009; Oliveira et al., 2012; Sass et al., 2012). Mulheres apresentam caraterísticas importantes na velhice, como viverem por mais tempo e mais sujeitas a doenças e problemas relacionados com a adaptação às mudanças fisiológicas da idade, tornando-as mais vulneráveis, incidindo em perdas psicológicas, sociais, como isolamento, viuvez e sintomas depressivos (Fernandes et al, 2009; Lima \& Bueno, 2009; Oliveira et al., 2012, Smith et al., 2015; Taylor, 2014).

Em relação ao fator idade e escolaridade, não se verificou associações estatisticamente significativas com a presença de sintomas depressivos nos idosos entrevistados. Esses resultados diferem de outros estudos, principalmente em relação à idade, onde indicam um aumento da prevalência de sintomas depressivos nas idades mais avançadas (Borges et al., 2013; Fernandes et al., 2009; Lima et al., 2009; Sasset al., 2012). A baixa escolaridade parece estar associada a maiores índices de sintomas depressivos, embora não haja consenso (Oliveira et al., 2012). É possível argumentar que os sintomas depressivos em idosos podem estar mais relacionados às condições socioeconômicas, culturais e aos aspectos biológicos do que apenas à escolaridade. 
No que se refere ao arranjo familiar, observa-se que os idosos que moram sozinhos, quando comparados aos idosos que moram com seus familiares e/ou cuidador, apresentaram diferenças significativas, indicando a presença de sintomas depressivos, com a percepção de uma visão de si e do futuro negativas. Morar só resulta na redução do suporte e trocas sociais e pode levar a sentimento de vazio emocional e solidão, predispondo o aparecimento ou aumento de sintomas depressivos (Fernandes et al., 2009; Sass et al., 2012; Oliveira et al. 2012; Smith et al., 2015; Unützer \& Park, 2012).

A percepção de uma visão de si e do futuro negativas dizem respeito a tríade cognitiva presente nos quadros depressivos, conforme descrita por Beck, Rush, Shaw e Emery (1997). Essas percepções condizem com padrões cognitivos que induzem ao indivíduo a ter uma visão negativa de si mesmo, percebendo-se como inadequado, indesejado e incapaz; e uma visão negativa do futuro, com a crença de que as dificuldades atuais continuarão indefinidamente, com dificuldades, frustrações e privações incessantes. Diante do exposto, chama-se a atenção para a solidão no idoso e a diminuição de seus laços sociais como um fator importante na relação com sintomas depressivos e na ocorrência desse padrão cognitivo com uma percepção negativa. Estudos futuros, que permitam comparar o desempenho do LIS-D entre população idosa e mais jovem, permitirão identificar se há diferenças entre a tríade cognitiva nessas diferentes etapas desenvolvimentais.

A presença de sintomas depressivos também está associada com dependência funcional e prejuízos cognitivos apresentados pelos idosos. Os idosos com alguma incapacidade funcional apresentaram sintomas depressivos e uma visão de si, do mundo e do futuro mais pessimista. A relação entre dependência funcional e sintomas depressivos também foi encontrada em diferentes estudos (Bretanha et al., 2015; Borges et al., 2013; Hoffman et al., 2010; Lima et al., 2009; Sass et al., 2012). A perda da autonomia e limitaçóes na vida diária geram um desequilíbrio nas emoções, no bem-estar e na imagem social, podendo desencadear os sintomas depressivos (Batistone \& Neri, 2007; Bretanha et al., 2015).

A relação entre déficits cognitivos e sintomas depressivos, encontrada nos idosos entrevistados, também foi observada em outros estudos (Alvarenga et al., 2010;
Borges et al., 2013; Lima et al., 2009; Sass et al., 2010). Já foi observada associação de sintomas depressivos e déficit cognitivo em idosos, estando inter-relacionados, pois os sintomas depressivos podem muitas vezes levar a quadros clínicos como demência (Lima et al., 2009). Transtornos depressivos em idosos têm sido considerados um fator de risco para o desenvolvimento de processo demencial. A comorbidade nesses quadros pode gerar comprometimento das capacidades funcionais, e as alterações cognitivas associadas a sintomas depressivos interferem no diagnóstico diferencial, especialmente nos quadros demenciais (Coutinho et al., 2016).

Foi identificada a presença de sintomas depressivos em idosos entrevistados, sendo maior a ocorrência de sintomas nas mulheres. Os resultados também indicam morar sozinho, maior dependência para a realização das AVDs e déficits cognitivos como fatores relacionados a sintomas depressivos nos idosos entrevistados. Ainda, houve correlações significativas entre a escala CES-D e o instrumento em validação LIS-D. O conhecimento dos fatores associados aos sintomas depressivos pode direcionar ações de manutenção da autonomia, da capacidade funcional e cognitiva, melhor suporte social e cuidados a saúde em geral que promovam uma melhor qualidade de vida a população idosa.

Considerando o aumento significativo da população idosa no Brasil, é necessário identificar e compreender os fatores associados a sintomas depressivos em idosos. No contexto da atenção básica, deve-se primeiramente atentar para a importância de estudos que busquem identificar e avaliar os sintomas depressivos na população idosa atendida, para que, a partir destes, possam ser efetivadas ações de tratamento e acompanhamento mais eficazes aos idosos acometidos pelos transtornos depressivos.

Estudos com instrumentos para avaliar sintomas depressivos e envelhecimento são fundamentais para uma melhor compreensão dos sintomas depressivos. As correlações significativas e positivas entre o CES-D e o LIS-D apontam para a validade de convergência dos instrumentos na aferição quanto a presença e intensidade dos sintomas depressivos nos idosos pesquisados. Considerando que o LIS-D é um instrumento psicológico em construção, esses resultados sinalizam para a validade de seu uso também com idosos.

\section{Referências}

Alvarenga, M. R. M., Oliveira, M. A. C., Faccenda, O., Cerchiari, E. A. N. C., \& Amendola, F. (2010). Sintomas depressivos em idosos assistidos pela estratégia saúde da família. Cogitare Enfermagem, 15(2), 217-24. doi: 10.5380/ce.v15i2.17850

Batistone, S. S. T., \& Neri, A. L. (2007). Percepção de classe social entre idosos e suas relações com aspectos emocionais do envelhecimento. Psicologia em Pesquisa, 1(2), 03-10. Recuperado de https://bit.ly/2DC4r10

Batistoni, S. S. T., Néri, A. L., \& Cupertino, A. P. (2010). Validade e confiabilidade da versão brasileira da Center for Epidemiological Scale - Depression (CES-D) em idosos brasileiros. Psico-USF, 15(1), 13-22. doi: 10.1590/S1413-82712010000100003 
Beck, A. T., Rush, A. J., Shaw, B. F., \& Emery, G. (1997). Terapia Cognitiva da depressão. Trad. Sandra Costa, Porto Alegre: Artmed.

Bertolucci, P. H. F., Brucki, S. M. D., Campacci, S. R., \& Juliano, Y. (1994). O Miniexame do estado mental em uma população geral: Impacto da escolaridade. Arquivos de Neuro-Psiquiatria, 52(1) 1-7. Recuperado de http://www.scielo.br/pdf/anp/v52n1/01.pdf

Brasil. Instituto Brasileiro de Geografia e Estatística. (2002). Perfil dos idosos responsáveis pelos domicílios no Brasil: 2000 . Rio de Janeiro.

Bretanha, A. F., Facchini, L. A., Nunes, B. P., Munhoz, T. N, Tomasi, E., \& Thumé, E. (2015). Sintomas depressivos em idosos residentes em áreas de abrangência das Unidades Básicas de Saúde da zona urbana de Bagé, RS. Revista Brasileira de Epidemiologia, 18(1), 1-12. doi: 10.1590/1980-5497201500010001

Brucki, S. M. D. I., Nitrini, R., Caramelli, P., Bertolucci, P., \& Okamoto, I.H. (2003). Sugestões para o uso do Mini-exame do Estado Mental no Brasil. Arquivos de Neuro-Psiquiatria, 61(3B), 777-781. doi: 10.1590/S0004-282X2003000500014

Borges, L. J., Benedetti, T. B., Xavier, A. J., \& D’Orsi, E. (2013). Fatores associados aos sintomas depressivos em idosos: Estudo EpiFloripa. Revista de Saúde Pública, 47(4), 701-710. doi: 10.1590/S0034-8910.2013047003844

Cavalcante, F. G., Minayo, M. C. S., \& Mangas, R. M. N. (2013). Diferentes faces da depressão no suicídio em idosos. Ciência E Saúde Coletiva, 18(10), 2985-2994. doi: 10.1590/S1413-81232013001000023

Coutinho, F. L., Hamdam, A. C., \& Baptista, M. N. (2016). Escala Baptista de Depressão para Idosos - EBADEP-ID: Evidências de validade. Perspectivas em Psicología, 13(2), 1-9. Recuperado de http://www.seadpsi.com.ar/revistas/index.php/pep/article/view/1

Dancey, C. P., \& Reidy, J. R. (2013). Estatística sem matemática para a psicologia. (5a ed.) Porto Alegre: Artmed.

Fernandes, M. G. M., Nascimento, N. F. S., \& Costa, K. N. F. M. (2010). Prevalência e determinantes de sintomas depressivos em idosos atendidos na atenção primária de saúde. Revista da Rede de Enfermagem do Nordeste, 11(1), 19-27. Recuperado dehttp://www.revistarene. ufc.br/vol11n1_html_site/a02v11n1.htm

Ferreira, V. R. T. (2012). Levantamento da Intensidade de Sintomas Depressivos - LIS-D. Passo Fundo: Manuscrito não publicado.

Hoffmann, E. J., Ribeiro, F, Farnese, M. J, \& Lima, E. W. V. (2010). Sintomas depressivos e fatores associados entre idosos residentes em uma comunidade no norte de Minas Gerais, Brasil. Jornal Brasileiro de Psiquiatria, 59(3), 190-197, 2010. doi: 10.1590/S004720852010000300004

Lima, L.C.V., \& Bueno, C.M.L.B. (2009). Envelhecimento e gênero: A vulnerabilidade de idosas no Brasil. Revista Saúde e Pesquisa, 2(2), 273-80. Recuperado de http://periodicos.unicesumar.edu.br/index.php/saudpesq/article/view/1173/792

Lima, M. T. R., Silva, R. S., \& Ramos, L. R. (2009). Fatores associados à sintomatologia depressiva numa coorte urbana de idosos. Jornal Brasileiro Psiquiatria, 58(1), 1-7. doi: 10.1590/S0047-20852009000100001

Lino, V. T. S., Pereira, S. R. M, Camacho, L. A., Ribeiro Filho, S. T., \& Buksman, S. (2008). Adaptação transcultural da Escala de Independência em Atividades da Vida Diária (Escala de Katz). Cadernos de Saúde Pública, 24(1), 103-112.doi: 10.1590/S0102-311X2008000100010

Oliveira, M. F., Bezerra, V. P. Silva, A. O., Alves, M. S. C. F, Moreira, M. A. S. P., \& Caldas, C. P. (2012). Sintomatologia de depressão autorreferida por idosos que vivem em comunidade. Ciência \& Saúde Coletiva, 17(8), 2191-2198. doi: 10.1590/S1413-81232012000800029

Organização Mundial de Saúde - OMS (2001). Relatório Mundial da Saúde. Saúde mental: nova concepção, nova esperança. Geneva, Suíça: Organização Mundial de Saúde.

Pinho, M. X., Custódio, O., \& Makdisse, M. (2009). Incidência de depressão e fatores associados em idosos residentes na comunidade: Revisão de literatura. Revista Brasileira de Geriatria e Gerontologia, 12(1), 123-140.doi:10.1590/1809-9823.2009120111

Sass, A., Gravena, A. A. F., Pilger, C., Mathias, T. A., \& Marcon, S.S. (2012). Depressão em idosos inscritos no Programa de Controle de hipertensão arterial e diabetes mellitus. Acta Paulista Enfermagem, 25(1), 80-85.doi:10.1590/S0103-21002012000100014

Silveira, D. X., \& Jorge, M. R. (1998). Propriedades psicométricas da escala de rastreamento populacional para depressão CES-D em populações clínica e não clínica de adolescentes e adultos jovens. Revista de Psiquiatria Clínica, 25(5), 251-261.

Smith, M., Haedtke, C., \& Shibley, D. (2015). Late life depression detection: An evidence-based guideline. Journal Gerontolical Nursing, 41(2), 18-25. doi:10.3928/00989134-20150115-99.

Taylor, W. D. (2014). Depression in the elderly. The New England Journal of Medicine, 371(13), 1228-1236. doi: 10.1056/NEJMcp1402180

Unützer, J., \& Park, M. (2012). Older adults with severe, treatment-resistant depression. The Journal of the American Medical Association, 308(9), 909-918. doi: 10.1001/2012.jama.10690

World Health Organization - WHO (2012). Depression: A global crisis: World Mental Health Day. Geneva, Switzerland: World Health Organization. October 10.

\section{Sobre os autores}

Claudia Daiane Trentin Lampert é psicóloga, Mestre em Envelhecimento Humano pela Universidade de Passo Fundo. Atualmente é docente do curso de Psicologia da Faculdade IMED.

Vinícius Renato Thomé Ferreira é psicólogo, Doutor em Psicologia pela Pontifícia Universidade Católica do Rio Grande do Sul. Atualmente é coordenador e docente do Programa de Pós-Graduação em Psicologia da Faculdade IMED. 